\section{The case for medical marijuana}

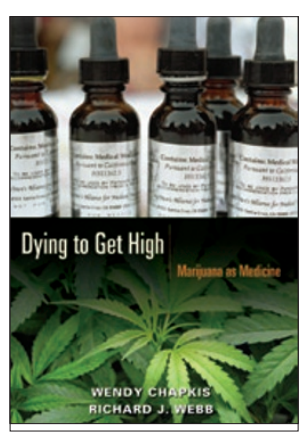

\section{Dying to Get High: Marijuana as Medicine}

\section{Wendy Chapkis and Richard Webb}

NYU Press, 2008

272 pp., paperback, $\$ 22.00$

ISBN: 0814716679

\section{Reviewed by Daniele Piomelli}

In 1996, a group of chronically and terminally ill patients in Santa Cruz, California, gave up on prescription medications and started using marijuana as a way to control the seizures, pain and depression caused by their conditions. They had varying personal experiences with the drugsome were old hippies, whereas others had never smoked pot before-but what brought them together was the conviction that marijuana, unlike other drugs, made their lives more livable. The group gathered around trauma patient and activist Valerie Leveroni-Corral to create the Wo/Men's Alliance for Medical Marijuana (WAMM), a nonprofit organization dedicated to providing cannabis to seriously ill people. Dying to Get High tells the remarkable story of this community.

With much empathy and a lively taste for detail, authors Wendy Chapkis and Richard Webb describe the difficulties involved in keeping afloat a cooperative like WAMM, such as the hard work of tending and harvesting the cannabis plants; the delicate task of transforming the plants into capsules, baked goods, tinctures and liniments; and the never-ending efforts to raise funds for the organization, which does not charge for its services and relies exclusively on contributions.

All of these activities are both labor intensive and technically demanding, and they must run smoothly on the backdrop of a legal environment that is anything but amicable. Even though California's Compassionate Use Act of 1996 permits patients to grow and possess marijuana with a physician's prescription, federal law still prohibits all such activities and considers cannabis and all of its chemical components (even those lacking psychotropic activity) to be as dangerous as the potent narcotic heroin. A 2005 Supreme Court ruling, which upheld the federal government's power to regulate the medicinal use of marijuana, further tightened this regulatory grip, exposing WAMM's members to new legal risks.

Given the toil and danger involved in growing and using marijuana as medicine, why don't the folks at WAMM simply ask their physicians for a prescription of dronabinol, an approved drug that contains $\Delta^{9}$-tetrahydrocannabinol ( $\Delta^{9}$-THC), the main active agent present in cannabis? This question lies at the center of the current debate on medical marijuana. Those who deny the therapeutic value of cannabis argue

Daniele Piomelli is at the Department of Pharmacology at the University of California, Irvine, in Irvine, California, USA and at the Unit of Drug Discovery and Development at the Italian Institute of Technology, Genoa, Italy.

e-mail: piomelli@uci.edu that the drug offers no benefit over existing medicines. They contend that the clinical acceptance of dronabinol-which is currently prescribed to treat the nausea and vomiting associated with cancer chemotherapy and to improve appetite in individuals taking HIV protease inhibitors- has rendered the use of marijuana obsolete.

Countering this argument, Chapkis and Webb report the testimonies of WAMM members who suggest that the beneficial effects of cannabis go beyond those recognized for $\Delta^{9}$-THC to include chronic pain, seizure activity, muscle spasms, anxiety and depression. For some medical conditions, such as pain, these personal experiences are corroborated by rigorous data. For example, a series of controlled clinical studies-supported by the Center for Medicinal Cannabis Research, at the University of California-San Diego- have provided strong evidence that smoked cannabis is both well tolerated by patients and superior to placebo in reducing symptoms of neuropathic pain. These clinical findings stand in contrast with the inconsistent analgesic effects generally shown by dronabinol and imply that there may be more to cannabis than just $\Delta^{9}$-THC.

This is not a new idea. Cannabis extracts contain a number of chemicals, some of which are known to have biological activities. For example, there is evidence that an analog of $\Delta^{9}$-THC found in cannabis called cannabidiol alleviates anxiety in people and reduces inflammatory responses and brain trauma in animals. When the body absorbs a cannabis extract, pharmacological interactions among these various components are likely to occur. Such interactions could explain why purified $\Delta^{9}$-THC does not exactly recapitulate the effects of marijuana. This is an interesting hypothesis, but it remains unproven.

Unfortunately, carried away by the same enthusiasm that makes Dying to Get High so readable, Chapkis and Webb take this idea for a fact, stretching it to create an image of cannabis that bears little resemblance to the real thing. Their fictive cannabis is an entirely "nontoxic herb" that through a rather mysterious "herbal synergy" maximizes the desirable actions of $\Delta^{9}$-THC while minimizing its untoward effects. The latter get little attention in the book, which only spends a dismissive remark on the addictive potential of the drug and does not mention the risk of psychosis that might be associated with its use by vulnerable individuals. This lack of balance is particularly distasteful because it is unjustified-the potential occurrence of these side effects cannot be used as an argument to deny medical marijuana to a terminally ill individual.

Not unexpectedly, a 'perfect natural remedy' such as cannabis finds its ideal counterpoint in the 'bad man-made drugs' that have been recently inspired by our increased knowledge of the cannabinoid system. Chapkis and Webb show no interest in these agents and no sympathy for the people who have devoted their lives to discover them. In fact, 15 years of research on cannabinoid receptors and their ligands go completely unnoticed in the pages of Dying to Get High. On this crucial issue, the book loses its steam to become a rant against the 'closedness' of modern medical science to new ideas, the greed of the pharmaceutical industry and the arrogance of federal agencies such as the US National Institute on Drug Abuse-all equally guilty of 'pharmaceuticalizing' marijuana. That's really too bad, because unless we set aside such simplistic schemes and start looking at cannabis for what it really is-a riddle in chemical biology and a broad opportunity for therapeutic discovery - the debate on its medical use will not move forward. 